\title{
Exploring the Roles, Practices and Service Delivery Mechanism of Health Service Providers Regarding TB in Two Urban Slums of Dhaka
}

\author{
Enamul Hasib ${ }^{1}$, Tariq-Ul-Hassan Khan ${ }^{2}$, Malabika Sarker ${ }^{1}$, Shayla Islam ${ }^{3}$, \\ Akramul Islam $^{3}$, Ashaque Husain ${ }^{4}$, Sabina Faiz Rashid ${ }^{1}$ \\ ${ }^{1}$ James P Grant School of Public Health, BRAC University, Dhaka, Bangladesh \\ ${ }^{2}$ Water Aid Bangladesh, Dhaka, Bangladesh \\ ${ }^{3}$ Health Nutrition and Population Programme, BRAC, Dhaka, Bangladesh \\ ${ }^{4}$ National Tuberculosis Control Program, Dhaka, Bangladesh \\ Email: enam.hasib@bracu.ac.bd
}

Received September 24 ${ }^{\text {th }}$, 2013; revised October $25^{\text {th }}$, 2013; accepted November $3^{\text {rd }}, 2013$

Copyright $(92013$ Enamul Hasib et al. This is an open access article distributed under the Creative Commons Attribution License, which permits unrestricted use, distribution, and reproduction in any medium, provided the original work is properly cited.

\begin{abstract}
Tuberculosis (TB) is a major health care burden in developing countries. With a high number of population living in an environment with high congestion, controlling TB in Bangladesh especially in urban areas has been a big challenge. The current study aims to identify the perception and treatment practice of formal and informal health service providers regarding TB in terms of treatment, referral system and to find out the partnership mechanism and also community perception and their health seeking behavior in two urban slums of Dhaka city. This is a cross-sectional study utilizing mixed methods approach and was conducted in two urban slums, namely, Slum A and Slum B of Dhaka city. Health service providers both formal and informal, community people and TB patients were selected as study population. In the quantitative part a survey was carried out where all the existing health service providers were interviewed. These health service providers were identified through 12 PRA (Participatory Rapid Appraisal) Social Mapping. Seven Focus Group Discussions (FGD) were conducted with this community. Popular Health service providers were identified through PRA matrix ranking during the FGDs and were selected for in-depth interview. TB patients were identified during FGD for in-depth interview. Community in urban slums is well aware of the infectious, contagious characteristics of TB. However, the long duration of DOTS program has been a major cause of high rate of drop-out. Generally drug sellers, traditional healers, homeopath and allopath (MBBS) practitioners are the primary point of contact of TB patients. They know where to refer to diagnosis and treatment. The referral system based on informal relationship sometimes leads to referring patients to wrong service providers. The mechanisms of TB programs in urban areas of Bangladesh should seriously consider arranging regular training and monitoring of health workforce, setting up formal partnership between formal and informal health service providers and generate information that policy-makers could use to scale up TB control program.
\end{abstract}

Keywords: Tuberculosis; Health Service Provider; Partnership

\section{Introduction}

Tuberculosis (TB) is a major health care burden in developing countries (Ravichandran, 2004). It is reported as the second highest cause of death from infectious disease after HIV/AIDS in the world. Incidence of TB cases has steadily declined in western and central Europe, North and South America, and the Middle East. But there has been striking unexpected increase in countries of the former Soviet Union, South Asia and in SubSaharan Africa (Frieden, Sterling, Munsiff, \& Dye, 2003). Although, Sub-Saharan Africa has the highest incidence rate (290 per 100,000 population), the majority of patients with TB live in Bangladesh, China, India, Indonesia and Pakistan where it is concentrated among densely populated areas (Dye, 2006). TB is a major health concern in Bangladesh. Even though, it is a curable disease, it has the highest rate of adult mortality in Bang- ladesh. The urban population of Bangladesh is growing at an annual rate of $5 \%-6 \%$ which soon will be $40 \%$ of the total population. The population of Dhaka city is approximately 12 million (Banglapedia, 2006) and about 30\% of the population live in slums and temporary squatter settlements without any access to basic primary health care services ((DGHS, NTP, 2009; ICDDR, B WP 142, 2000). Slums are vulnerable due to poor living conditions and are not recognized by government as "legal settlers". The overcrowding, poor ventilation and unhygienic living conditions in slums favor the spread of TB. David et al. (2007) found that children living in a squatter settlement are 9 times more likely to have TB than non squatter children in one of the Asian developing countries. David et al. (2007) also described that gender is major cross cutting determinant of health inequality. Poor urban women generally exhibit higher 
risks for diseases and poorer overall health status than poor urban men (David et al., 2007). According to the TB case notification report submitted to the $\mathrm{WHO}$, about twice as many men as women were notified as TB patients (WHO, 2008). The prevalence of $\mathrm{TB}$ is comparable between males and females until the age of 15 years, and then disparity starts. But TB progresses from infection to active disease much faster in women during their reproductive ages than in similar age groups of men. The gender differences in behavior may influence disease progression (Karim, 2009).

NTP notified a total of 151,062 of all forms of TB cases (104/100000 population) nationwide. $17.7 \%$ of them were reported from urban areas. According to the WHO Report 2005, a total of 7556 new smear positive, 1134 relapse, 4986 smear negative pulmonary, and 2689 extra-pulmonary TB cases were found and a treatment success rate of $76 \%$ was achieved in the metropolitan areas (Dhaka, Chittagong, Khulna, \& Rajshahi) which was $15 \%$ lower than that of the upazilas (91\%). Detection rates are particularly problematic among urban poor women living in slum settlements and there are a large number of TB patients who seek treatment from private sector and informal providers. It is important to acknowledge that most private sector health personnel and informal providers are not officially part of the formal government health system, and therefore do not follow NTP guidelines. This can reduce the efficacy of TB services received by patients and discourage the accuracy of NTP statistics on the TB situation, with an increased potential for drug-resistant TB in Bangladesh (TB Policy in Bangladesh, Public Health Watch, 2006). There have been informal attempts for partnerships between the government, NGO sector and informal/private sector, but coordinated effort to create a national implementation strategy for engaging private providers is absent (WHO, 2010). One challenge faced by the partnership is balancing the needs for central level control and local responsiveness. Most of the time, decision making at the central level creates delay in endorsing local level activities formally. In this context, encouraging local staff to work with NGOs to solve problem at the local level can be difficult (Islam et al., 2011). The multiplicity of providers, fragmented services, absence of appropriate coordination partners, and insufficient coverage lead to gap and duplication in treatment leaving the urban poor grossly underserved. Apart from partnership, the challenge also lies in communication and community initiatives for referral of suspects for case detection in the urban areas (Islam et al., 2011). Informal partnership and relationship among the local level informal health service providers in urban slums of Dhaka has not been discussed much in previous studies and hence, a gap in literature is found which created the scope of this study.

The current study aims to find out the partnership mechanism among the range of formal and informal health services providers and to explore health seeking behavior of slum communities suffering from TB.

The government of Bangladesh has shown its commitment to control TB by establishing the National Tuberculosis Control Programme (NTP). In 1994, BRAC and the National Tuberculosis Program (NTP) formalized their relationship to enable coordination on a large scale in anti-TB care. The Global Funds to Fight AIDS, TB and Malaria (GFATM) project has helped smaller local NGOs to offer TB control services (model of community-based DOTS) all over the country in an effective and cost-effective manner (TB Policy in Bangladesh, Public
Health Watch, 2006). Launched 2002, the Global Fund to Fight AIDS, TB, and Malaria (Global Fund) represents a surge in dedicated resources for TB. In 2004, the national partnership for TB control included 10 NGOs, including BRAC, implementing TB services. In 2011, there are 44 institutions working on TB control, including several non-implementing partners that provide technical support and operational research (Bangladesh CCM, 2009: 41). The DOTS coverage by BRAC is more intense and organized in rural areas than the urban areas. Because many urban patients sought care from private practitioners or drug sellers, BRAC reached out to these private practitioners and provided education about TB diagnosis and treatment and encouraged referrals of TB patients to BRAC and government facilities. By the end of 2007, according to Islam et al., (2011) BRAC covered a catchment area of 3.2 million in Dhaka, including those covered by the 18 peri-urban centers, and had diagnosed over 3100 cases of TB.

\section{Methodology}

\section{Study Design}

This is a cross-sectional study utilizing mixed methods approach. The study has three components, first: a social mapping of health providers to identify the providers' engage in providing TB treatment or referral, second: a quantitative survey of the health providers identified through social mapping, and third: Focus Group Discussion (FGD) with community and qualitative interview with health providers, key informants and clients. Through 12 social mapping with the community people, 53 service providers including both formal and informal who are providing anti-tuberculosis care were identified and were taken study population for the quantitative survey. Later focus group discussions and in-depth interviews were conducted as part of qualitative component. In-depth interviews were carried out with the TB patients were identified in the FGDs. 7 FGDs with both men and women were carried out and 24 TB patients were interviewed. The criteria for selecting TB patients was people who have been diagnosed as TB, currently under any form of TB treatment and/or have discontinued treatment but not cured. Popular service providers were also identified in the focus groups discussions and social mapping by community groups, and were selected for in-depth interviews. 27 popular health service providers were interviewed.

\section{Study Site}

BRAC provides TB related health care services in the periurban area through BRAC Health Program and in the urban slums through partner organizations. Two slums were selected purposively in consultation with BRAC TB program; as study sites based on long history of existence, performance in terms of TB case notification rate (one selected slum is comparatively low performing and another one is comparatively high performing) and presence of long term interventions by BRAC and partner organizations.

NGO operating in Slum A is NGO A and NGO B in Slum B. The estimated total number of households in Slum A is 4098 with a total population of 16,380 (based on discussion with NGO A and slum dwellers). Total household at Slum B is 2170 and population size 9350 (based on discussion with NGO B and slum dwellers). 


\section{Data Collection}

Social mapping was carried out to identify the existing health service providers. Slum A is divided into five parts and the boundaries of the parts were identified with the help of the slum dwellers. A total of 8 social mappings were done in Slum A. Four social maps were drawn in Slum B. The participants of the social mapping were asked to draw the geographical map of the area. In every session one person from the participants was identified to draw the map. They were asked to identify the boundary of the respective area, roads, water sanitation facility, bazaar, educational institute and place of practice and the catchment area of the health service providers active in this area. Thus a geographical map and a comprehensive list of health service providers were developed. During conducting social mapping, listing of all health service providers including both formal and informal who are delivering health services in the two selected slums were carried out. These providers range from qualified allopathic doctors gaining MBBS degree from different medical colleges, semi-qualified allopathic providers that include providers who have received training of varying duration from a formal institution in the public or private sector, drug sellers most of whom have had no training in dispensing drugs, traditional healers like Kabiraj whose practice is based on diet and herbs, faith healers mostly providing spiritual treatment etc. Apart from them there are NGO workers who provide DOTS in the urban slum area. Then the survey was conducted among them. The survey collected data on knowledge, role, treatment practice of TB, partnerships if any and referral linkages with other institutions/providers. Given that the number of providers (formal and informal) available was not too large, all providers listed were interviewed. A total of 29 service providers from Slum A and 24 from Slum B slum were interviewed. Focus group discussions (FGD) with community were conducted to understand perception, stigma, gender issues and the health seeking behavior regarding TB and popularity of health service providers. 7 FGDs were conducted, 3 at Slum A and 4 at Slum B. Four FGDs were conducted with men separately and 3 with women. In addition to asking communities to rank popular health service providers, causes for their popularity were also identified to understand reasons to access certain providers over others. The PRA ranking exercise was conducted in the following manner: participants were asked to number-1) the health service providers on the basis of their popularity and 2) the causes for their popularity separately in a scale of 1 to 10 (10 being the most popular and 1 being the least). Flip-charts were used for doing the rankings, note was taken and recorders were used for recording the discussion. Notes were taken in Bengali and the transcripts are written also in Bengali followed by English translation for analysis. After completing survey and FGD, in-depth interviews with selected health service providers were carried out. Twenty seven (16 from Slum A and 11 from Slum B) health service providers were selected purposively following a set criterion for in-depth interview and the criteria for selection were: popularity of the service provider among the community, experience on delivering health service in the locale and diversity of health service providers. In the in-depth interviews, questions were asked on knowledge and perception of TB, health service mechanism in terms of TB case detection, treatment and referral, and the process whereby they have local level partnership. Along with health service providers in-depth information from TB patients of these two areas were also recorded for triangulation. TB patients were identified during FGD. Criteria for selecting TB patients were active TB patients currently undergoing any form treatment, treatment drop-out and cured from the disease. A total of 24 (13 at Slum A and 11 at Slum B) TB patients were interviewed. All the notes were taken in Bengali and also the detailed transcripts were written in Bengali. Then these were translated into English for analysis

Before starting data collection, questionnaire and interview guidelines were pretested and piloted, and necessary revision and modification were carried out. The questions were standardized to ensure reliability, generalizability, and validity.

\section{Data Analysis}

Review and analysis of ongoing data found missing data in some case which required clarifications. So the team members went back to the field to collect more data, or repeated in-depth interviews for clarifications. This process of review, update and additional fieldwork was done throughout the research period. Transcribed qualitative data were translated into English and coded thematically for analysis. In every stage, quality was controlled by the senior research team and supervisors. Thus reliability and validity of data were ensured. Univariate and bivariate analyses of quantitative data were carried out to explore the findings of survey with health service providers regarding their background information, perception, knowledge, role, treatment practice, training, referral, local level partnership.

\section{Ethical Approval}

Written informed consent was taken from all participants before the interview took place. The informed consent form included all necessary information for the participants that was read out at the beginning of every interview. It was maintained strictly that there was no coercion during data collection. Participants were informed that they were absolutely free to withdraw from the study at any time even after giving their consent. It was also mentioned that there was no direct benefit for participants from the study. Confidentiality and anonymity of the TB patients were given supreme priority.

\section{Results and Discussion}

A variety of private and informal institutions have been implementing anti-tubercular care with differential effectiveness in urban slum area of Bangladesh. These providers are qualified medical doctors, allopathic practitioners who received short or long term training from a public/private formal institution, drug sellers most of whom have had no training in dispensing drugs, traditional healers like Kabiraj whose practice is based on diet and herbs, faith healers mostly providing spiritual treatment etc. Apart from them there are NGO workers who provide DOTS in the urban slum area. According to the survey conducted among the 53 health service providers, $77 \%$ were male and $23 \%$ respondents were female. Majority of the health service provider (93\%) had academic education while $7 \%$ had no academic education. More than two third (69\%) of the total respondents completed up to higher secondary education whereas, about one fifth (22.4\%) had graduate or post graduate level of education. $8.2 \%$ respondents passed from Madrasa (Arabic schooling system). Among the health service provider majority i.e. $43 \%$ were 
drug seller (Pharmacy), 25\% were NGO worker or Health Assistant or Midwife, $15 \%$ were homeopath, $7 \%$ were Spiritual Healer, 6\% were Allopathic, 2\% herbalist, 2\% were acre farm. About two third (66\%) of health service providers reported having medical qualification while remaining one-third (34\%) had no such qualification. Of them 51.4\% have RMP or PC or LMAF degree, 20\% have Paramedics or Midwife degree, $17 \%$ have Diploma or Diploma in Medical Assistants degree, 11\% have Homeopath degree, 3\% have MBBS, Medical Technologist degree.

\section{Knowledge and Perception of TB}

A number of varied beliefs and understandings on the causes and spread of TB existed among providers and community people in the two urban slums. These ranged from biomedical reasons, environment conditions, to supernatural causation, and social and economic factors. Gender, old age, mobility and life style and behavior were also seen to contribute to TB. Most of the service providers (informal and formal) reported that TB is an infectious disease caused by bacteria. The risk factor of the disease includes living in densely populated unhealthy and polluted environment. It is known as TB and "Jakkha” in Bengali. A drug seller of Slum A said, "If cough lasts for more than 2 - 3/4 weeks and doesn't cure, then it can turn into TB”. About half of the health service providers (49.1\%) reported about TB patient's coughs or sneezes as routes of transmitting TB followed by Unhygienic environment (Dust) (41.5\%), From infected (TB) person's breathing (30.2\%), getting cold/getting cold long time (13.2\%), polluted or dirty environment (5.7\%), density of people, damp environment (3.8\%) etc. Besides, respondents also reported smoking (43.4\%), genetic disorder/ inherited (24.5\%), alcohol/drug use (17\%), eating from the same plate $(11.3 \%)$ etc. as the causes of transmission of tuberculosis. A common understanding among the respondents was that this disease affected men more than women as they were more mobile, and ate street food. One TB patient, aged 24 year's male from Slum B shared, "If someone eats at restaurants, they can be affected by TB because a lot of people eat at restaurants and it is not possible to know who among them have TB and who have not. And thus TB can spread in restaurants while sharing same utensils". Both health providers and community people shared that life style factors such as smoking, drug addiction and injury caused in lungs from smoking tobacco could lead to TB. Lack of nutritious food in the body also played a role. Many health providers reported that by taking full course of TB drugs, keeping away from unhygienic environment, dirt and garbage one can prevent themselves from getting infected by TB. Many also mentioned "quitting smoking” as a way to prevent TB infection which is a wrong perception. A few of the respondents both providers and community people shared that TB was a hereditary illness and could not be avoided it if was in the family blood.

\section{Polluted and Congested Environments}

Many stated that TB occurred from staying in unhealthy and unhygienic environments. Slums were seen to be very vulnerable living spaces for the spread of TB. Slums tend to be densely populated areas, congested, with little sanitation and poor drainage systems in place which allow for the rapid spread of disease. Some of them shared that open defecation may also cause TB as this created an unhygienic environment. One participant living in Slum B shared, "If someone throws waste materials in front of house, the environment gets dirty. The occurrence of this disease is higher among the slum dwellers because they live a congested and dirty life, sometimes they take their meal sitting on the same place where they defecate. This is dirty environment". In these overcrowded and congested spaces, people stay close to TB patients, which is seen as vulnerability to the disease. Family members of a TB patient are very likely to be affected by TB because they live very closely; share bed, utensils, food; touch each other. Among the respondents from community people, who stated that, TB is a contagious disease; emphasized the importance of wearing masks and using separate utensils to prevent TB infection. But sometimes masks are not very available in the neighborhood shops, so that it is not always possible to wear mask and due to the poverty sometimes using separate utensils is not always possible either.

\section{Signs and Symptoms of TB}

The survey data highlight that most of the service providers (informal and formal) perceived that a persistent cough, which lasted more than 3 weeks was TB. Three fourths of them reported that cough that lasts longer than 3 weeks as signs and symptoms of TB followed by coughing up blood (60\%), fever/ fever at night/fever without clear reason persisting for more than 7 days (58.5\%). Fifty six percent said that cough/cough that lasts for more than 1 week was TB and $47.2 \%$ mentioned signs of weight loss, $32.1 \%$ mentioned ongoing fatigue/loss of appetite, $17 \%$ mentioned chest pains and $11.3 \%$ mentioned sweating at night. In the in-depth interviews and focus group discussions, providers as well as community people also shared similar types of perceptions on signs and symptoms, but a common understanding that differentiated from a simple cough, was a lingering cough. However, among the respondents there was a variation of knowledge in terms of number of days for coughing that ranged from 7 to 30 days. Other signs included, coughing up blood, losing weight, loss of appetite, suffering from fever for more than one week, weakness, throat irritation at initial stages etc. All the TB patients who were interviewed mentioned TB as a terrible but curable disease. They mentioned coughing and vomiting with blood, losing appetite and weight as the symptoms of TB. A TB patient from Slum A explained, "A TB patient loses his appetite (khudhamonda) so much that, even drinking water might cause vomiting". A TB patient start losing body weight at a very quick rate and starts feeling weak and sleepy all day. A TB patient from Slum A, 18 years of age, male said, "When someone gets $T B$, it is very likely that he/she will be affected with few other diseases like Jaundice, Typhoid, fever cold etc.".

\section{Stigma}

A number of respondents-both service providers and community people claimed that TB patients, irrespective of sex are usually avoided and treated badly. In some cases, both providers and community people shared stories of women who were abandoned by their husbands because of TB or some women remain unmarriageable because they had suffered from TB. There were stories about TB patients being isolated, discouraging them from going to social gatherings, market places and 
deliberately avoiding any interactions with them etc. In some extreme cases, it was reported that some families ended their relationship with the patients. However, there was also a narrative of a woman who was suffering from TB and her husband was extremely supportive and didn't abandon her. A few respondents claimed that women were more vulnerable compared to male patients, because of their lack of economic and social power and they didn't have control over when they could seek treatment. According to many, in the past it was believed that a TB patient was suffering because of their own "sin and curse" which often placed a moral judgment on the person, but this was slowly being replaced by blaming external factors, which didn't blame the individual for the illness. A male TB patient from Slum B explained, “No, TB is not caused by having sin or being cursed. Anyone can be affected by TB. However, people who smoke biri-cigarettes have the greatest possibility of getting the disease. Besides, those who have a reckless lifestyle, live in dirty surroundings, take addictive substances or drugs may also get TB". However, despite these changes, fears and superstitious beliefs continue. One respondent, 40 years old male TB patient from Slum B said TB could spread by showing hatred towards a TB patient. He described, "Once I saw a woman in front of Ajimpur Maternity. She coughed up blood through nose and throat. Watching her I felt abhorrence. After one week of that incident I got affected by that disease. I watched that woman from a distance. I didn't even touch her. But I got affected by tuberculosis". Many of the respondents said that gender discrimination and stigma existed in the past when they were younger and living in village but these were less frequent in urban areas. However few of the female respondents shared some of their experience regarding gender and stigma. One female TB patient, 30 years of age from Slum B shared, "When I had TB, my neighbors did not come to my house, and I did not visit anyone. I spent most of the time in my room, on my bed, and I felt depressed, as I felt that no one came to my room because of my illness. Sometimes I thought I have not committed any sin, so why did this happen to me?" Interviews with women and men and providers found that in many cases, females tend to hide their disease. They tolerate fever, cough, pain until these become severe, as many cannot afford to pay for treatment and often are too scared to seek treatment. In addition, it was shared by both community people and providers that females who had suffered from TB in the past still faced challenges in getting married. A male drug seller shared, "After proposing for marriage, if groom's relatives come to know that the bride suffered from tuberculosis earlier then they don't show further interest. They start looking for another bride. They think that she may get affected in the future too". Health service providers argued that since treatment was widely available now and people could be cured there was no point in avoiding TB patients, keeping them isolated and canceling marriage proposals etc.

\section{Health Services}

The health care service in terms of TB in both Slum A and Slum B involves providers like pharmacy based drug sellers, MBBS doctors and paramedics, homeopath doctors, spiritual healers, ayurveda and traditional healers, NGOs active in these areas, diagnostic centers, government hospitals etc. Initially patients come with the complaints of fever and cough. Most of the patients are residents of the slum. They prescribe them anti py- retic and anti histamine drugs and sometimes antibiotics. If the fever and cough are not subsided, moreover patient start losing weights, then they take different approaches depending on their type of practices. This finding is also evident in the survey data. One-fifth of the health service providers provide case detection (diagnosis of TB) service to the suspected TB positive cases. Majority i.e. $83 \%$ of the health service provider reported that they usually refer the suspected or diagnosed TB patients after treating the patient for 2 - 3 weeks. Survey data revealed that $13 \%$ health service providers do not provide any TB related health service. The initial treatment is given according to the symptom of the patient. When these problems are not reduced then the patients are referred to diagnostic center, hospital for lab diagnosis of TB. Usually allopathic doctors either send them to diagnostic centre for further evaluation or directly to health facilities like TB hospitals or NGOs providing services for diagnosis and treatment of TB. Drug sellers also refer patients to government hospitals or NGOs. NGO workers visit door to door and inform people about satellite and static clinic. If they find anyone with symptoms of TB or any diagnosed TB patients, they send him/her to satellite clinic. Then depending on diagnosis, patient is enrolled for treatment of TB. NGOs also receive patients referred by the local health service providers be it allopathic doctor or drug seller. Once a patient who goes to a NGO clinic, he get himself registered sees a doctor. If the patient is diagnosed with TB, he has to fill out a TB patient form and being given a card for collection of medicine. For laboratory tests, poor patients are referred to government facilities and those who can afford are referred to private clinics. According to the service providers, patient prefer to go to private diagnostic centers as they don't have to wait long and they receive proper service. Finally, it depends on the financial condition of the patient whether he will go to a government hospital or a private facility. Mainly patients are sent there for cough test and chest X-ray. In some cases, blood test (Mantoux Test) is done. Homeopath doctors sometimes continue their treatment even after the patient is diagnosed as TB patient. In some cases, they claim, that patient came round. But when the treatment fails and condition of patient keeps deteriorating, they refer patients either to a government hospital or an NGO providing treatment for TB. In that case, the patient is already in the advanced stage. One of the respondents quoted, "After taking medicine for 90 days, cough test, blood test and X-ray are done. If germ of TB is still identified then I provide a drug for 15 days. Even after that if patients don't get well, I suggest them to receive treatment from TB Hospital”.

\section{Health Seeking Behavior}

Primarily people in the slum seek treatment from the pharmacy close to their residence or workplace when they have fever, cough, and cold. Besides taking medicine from the pharmacy, they also go through some home remedy like for fever, they pour water on their head, wipe their body with wet cloths; for cold, they take hot tea etc. while choosing health service providers, people consider the closeness of the health service providers from their residence, popularity, cost of treatment etc. sometimes they choose the health service providers by their own and sometimes they are referred by neighbor or relative who have experience of seeking health service in the locality. One TB patient from Slum A who is 35 years old male said, "In the beginning I had light coughing and I thought the coughing 
was caused by the hot weather. The coughing started to increase slowly and continued for about one week. Then I felt that I was suffering from fever and went to the pharmacy near the gate of Niketon. As it is close to my house I go there for health service for any minor health problems. I brought medicine for fever and coughing and took medicine for about 15 days. But when I was not getting well, I went to the TB hospital according to the advice of my brother". When the cough, fever, cold are not cured by taking medicine from local facilities people seek for other health facilities. Cost of treatment plays a vital role in choosing health care service. People were mostly found to be more relying on government hospital like TB Hospital comparing to NGO facilities regarding TB treatment and the local pharmacies also refer most of the TB patient to government hospitals rather than NGO facilities. The perception of incurableness of TB has been changed probably due to more and more availability of DOTS, treatment success and increased awareness among the community. Respondents now believe that a TB patient can completely come round if s/he takes full course of medicine in right time from the Family Health Centre or the City Health Centre, Surjer hasi Clinic (CWFD), UTPS, BRAC Health Centre, TB hospital at Mohakhali. One 18 years old male TB patient from Slum A said, "The doctor prescribes medicine according to the condition of the patient. If the condition is severe, s/he might have to take medicine for 6 months. If it's less severe, then 4 months and if it is the least severe, then 2 to 3 months of medicine can cure tuberculosis".

\section{Referral System}

Near about three fourth (72\%) health service providers reported that they refer TB patients to a government health facility. In Slum B 46\% health service providers noted that they refer diagnosed or suspected TB patient to CWFD clinic DOTS center while in Slum A 25\% health service providers refer TB patients to UTPS. Besides $10.9 \%$ health service providers refer TB patients to a NGO health facility followed by $2.2 \%$ to MBBS Doctor, 2.2\% to Homeopath Practitioner. However, the service providers at Slum A, also prefer to send their at BRAC health facility Moddho Badda' which is far from the slum. The reason for preferring BRAC health facility is that there one doesn't have to be a victim of peddlers. The standard of treatment is good here and all the treatment and medicine are given free of cost. The caretakers come to the house of the patients and make them take the medicine. TB hospital falls short to BRAC health facility because people are reluctant to go to TB hospital as they have to pay extra money to the staffs. Peddlers are very active there. People do not want to go through the hectic process the hospital. Female patients are also reluctant to go to hospital as once they go there their physical conditions will be revealed and will be known to many people. At the same time, this is also true that TB hospital is an old institution, a specialized institution for TB and well-known as it is close to the neighbourhood. Many people prefer to go there as the communication facilities is easier. Then again often the patients have familiar staffs at the TB hospital. Many people do not know about BRAC health facility or the UTPS. In Slum B, TB symptomatic patients are being referred to TB hospital in Mohakhali and Shyamoli, Sohrawardi hospital, and Smiling Sun clinic of CWFD. Smiling Sun clinic is much preferred option as most of the patients are being referred here. Smiling Sun visits slum regularly. They arrange satellite clinic at slum on every Wednesday. Sometimes people go to TB hospital at Mohakhali for treatment. Later they are referred to Smiling Sun clinic because it is within the reach of the people of this area. One NGO worker from Slum B mentioned, "Having treatment is easy. If anybody can reach up to treatment centers, medicine is given free which is a big opportunity for poor patients. All they have to do is to go to the facility". TB hospital is also in demand in Slum B as this is a specialized hospital for tuberculosis. Having reference from TB Hospital makes it easier to have medicine from Surjer Hasi.

\section{Follow-Up}

Private services providers do not keep track of their patients, as they do not have such system. However, since most of the service providers live in the neighborhood, they know the patients personally. Some of the health service providers try to keep track of the status of their referred patients informally. If they visit a patient on call and if there is a TB patient in the neighborhood, they visit him as well. Thus, service providers stay connected with the patients. One of the drug sellers from Slum A, aged 26 said, "As most of the patients are from this area, I meet some of those patients when I move around". This connectivity sometimes helps them to identify patients who do not complete treatment or course of medication. As the treatment is long term, patients become reluctant to take medication once they start getting well. Generally, women tend to complete the treatment than men. They try to cure fast by taking drugs regularly. Sometimes patients leave hospital on "risk bond" because it becomes difficult for them to stay at hospital for three months. Many patients come from village and intend to stay for 5 - 10 days. This affects their treatment a lot. Some of these patients are identified by the service providers in the community and also by the NGOs. NGO clinics keep track of their patients through field staff. They make sure that all the patients are taking drugs properly and regularly. If someone leaves the city and goes anywhere else then the patient is issued a card so that he can receive medicine from the local health care center.

\section{Challenges in Seeking Treatment}

One of the common challenges found during receiving treatment for TB was the lengthy nature of the treatment. Many people start the treatment but stop it at the middle of the treatment. One respondent, a TB patient of age 35 years from Slum A who stopped taking medicine before completing the course and didn't resume the treatment explained, "They told me to make the documents again. I work at garments factory. If I would go for new documents, I had to spend 3 - 4 days and had to miss office for those days. Salary for those days would be deducted. That's why I didn't go for new documents". TB patients have to go to Smiling Sun to collect the medicine every day or twice a week which sometimes cause problem for them to maintain regularly. Another male responded, a TB patient, 57 years of age from Slum A explained another problem that he faced during taking TB treatment from UTPS, "The problem that I faced is that I have to go daily to collect my medicine. UTPS doesn't give medicine in one lot. The biggest problem is that there is no one there to administer the injections. Every day I have to pay tk. 10 to a pharmacist elsewhere to get my injection. If I miss an injection, I will be finished. If UTPS 
would push the injections, then I wouldn't face this problem". Patients also discontinue treatment when they start feeling better after taking medicine for few days. At one point they stop taking medicine and eventually start suffering from TB again and have to start treatment over again.

\section{Partnership}

Most of the service providers especially pharmacy based drug sellers, private doctors, traditional and spiritual healers do not have any formal partnership neither among themselves nor with the hospitals and NGOs active in Slum A and Slum B area. But in terms of informal partnership (which is more like a relationship than partnership), many of them maintain linkage with other private service providers, diagnostic centers, NGOs and employees of hospitals. Basically this partnership develops because of long term kinship, accessibility and presence in the neighborhood. This informal partnership also develops between individual service providers and NGOs and hospitals. At Slum A, many service providers send patients to UTPS or TB hospital. Personal relationship with employees of NGO and hospital plays role in such case. Patients also get benefit from this arrangement as hospital employees take special care if patients mention about a private service provider whom the hospital employee knows well. One homeopath practitioner from Slum A said, "I send patients to Dr. Borkotullah. If patients take my name to him then he pays more attention. In case the patient is poor, sometimes he provides sample drug if it's available". Another reason for sending patients to NGOs is that they may get selected for training. This informal partnership mostly does not involve any financial benefit unless this is with any commercial diagnostic centers or private clinic. Sometimes partnership develops only because of the goodwill of the provider. Drug sellers at the pharmacy send patients to NGOs active in their neighborhood. Field workers form NGOs also visit pharmacy, private doctors and request them to send patients who have TB symptoms there. They also inform service providers about treatment of TB and inform them that cough tests are free of cost. They give them leaflets of their organizations. Sometimes private service providers send patients to diagnostic centers for cough test instead of sending them to some NGOs where cough test is free. The reason behind this is they get financial benefit by sending them to a commercial facility. Working in partnerships can improve appropriate and timely referral system among the service providers. On young allopath practitioner from Slum B mentioned, "I passed MBBS in 2007. I have been working here for the last five years and now realize that if we all work together, we will be surely successful. Drugs and tests are free but we have only one problem; we have been failed to make our management system efficient".

\section{The Role of NGO and Other Actors in Preventing TB}

NGO and service delivery organizations promote timely access and treatment of TB, and the use of face masks among TB patients to avoid the spread of infections. However, this outward use of mask was challenging for many of the patients, as they were made fun of and it was an obvious "marker" of being an infected patient. According to the respondents both health providers and TB patients, this happened due to superstitions, lack of education and social awareness. They argued that the situation could be improved if staffs from the government hospital, members of the community, NGOs like BRAC, and doc- tors and nurses come forward to speak freely on TB, which contribute to promoting understanding and awareness, remove stigma and create sympathy for patients. According to a homeopath practitioner from Slum A, "TB is a completely curable disease. People in the government hospital, members of the community, NGOs, doctors, nurses, BRAC should come forward to educate people". Health service providers think active social awareness campaigns need to be created to prevent TB. Local doctors, pharmacists, elected public representatives. Imams of the mosques should work together to create awareness among the community.

\section{Training}

Only one fourth of the health service providers had received training on TB related issues while the remaining three fourth did not receive such training. Among those who received training on TB more than half (54\%) received refresher training. Majority of the participants (83\%) reported about further needs of training on TB. BRAC organizes training on TB for the NGOs like UTPS, CWFD etc. through whom the service is being provided. UTPS in Slum A area and CWFD through smiling sun franchise in Slum B area provide trainings on TB to the health service providers such as MBBS doctors, RMPs (rural medical practitioners), diploma holders, persons with allopathic and homeopathic backgrounds, medical technologists, pharmacy based drug sellers, and other related people in the community, i.e. religious leader, community leaders. NGO workers who are involved in case detection and treatment of TB are also given training. Basically service providers who are active in these areas for a long duration of time are approached and selected for training. Often newer service providers are left out. Sometimes participants are selected through personal connection. One drug seller from Slum B quoted, "I send them patients, they give me the opportunity to participate in the trainings". These trainings focus topics like causes of TB, signs and symptoms of TB, treatments, preventive measures, people vulnerable for the disease etc. Besides, options for counseling for the positive patients, correct way of taking the drug, symptoms after starting medication are also discussed. Most of the service providers found these trainings very useful. Through trainings they acquire knowledge regarding not only the disease itself and its treatment but also an idea about where to refer TB suspect patients. That's why service providers who do not treat TB thought that this kind of trainings can be very useful for them also. So, if they can identify the disease and refer the patient properly that will decrease delay in treatment significantly. Beside this, they can play active role in the community in terms of providing correct information. Unfortunately, most of these service providers noted that they did not attend any refresher's training or any intermediate training. It's very difficult for the service providers to update and upgrade their knowledge if there is no refresher's and intermediate level training.

\section{Recommendation from Health Service Providers}

Health service providers think that it is very important to build awareness about TB among both health service providers and patients. One of the ways is to organize campaigns by the government including all service providers, imams of the mosques, school teachers. They should be given training to raise awareness of the community regarding prevention and 
treatment of TB. One drug seller quoted, "People must be made aware. Those who do not know much about TB should be informed. Those of us who are aware should make others aware". Sex workers and drug users must be aware of the problem. Sewerage system of the slum areas needs to be improved. All these need to be organized in a coordinated way. For example, mostly people are at work during weekdays. So, any training and awareness program should be run during weekends to ensure maximum participation from the community. Health check-ups must be conducted in every 3 - 4 months. There should be special emphasis on education in the slum area. In fact, a special committee can be formed in each ward that would take initiative to bring patients with symptoms of TB under treatment. Activities and coverage of NGOs need to be increased. Organizations like BRAC should take initiatives in this regard. A coordinated effort is required to eliminate TB. "Patients will get proper treatment if all organizations think the same way".

\section{Conclusion}

Controlling TB is a tough challenge for countries like Bangladesh. However, BRAC's approach to controlling TB through DOTS in Bangladesh has achieved significant success by employing public private partnership. BRAC is the leading NGO working with NTP for controlling TB, and it has also achieved significant result. But in terms of urban areas, controlling TB still remains a big challenge because of high population density, unhealthy living environment, lack of necessary services and also migratory characteristics of people. Interestingly, community people are well aware of the infectious, contagious characteristics of TB which is absolutely curable by proper medication. They have been gradually free from the misconception, stigma and superstition related to TB. The problem they face is living in unhygienic condition which is conducive for transmission of the disease. They cannot avert this situation. Regarding the health seeking behavior, people of urban slum mostly come in contact of health service providers in the early stage of the disease. However, major problem in terms of TB lies with completion of treatment. The long duration of DOTS program was found to be the reason behind this. Also less reporting of female patients still remains as a major concern. Diverse categories of health service providers were found in the study area who provide treatment for patient with cough and fever for a certain period of time and then they refer the patient to other facilities if they fail to cure cough and fever. Generally drug sellers, traditional healers, homeopath allopath (MBBS) practitioners etc. are the primary point of contact of TB patients. As a result of frequent trainings organized by NGOs and the government, even less qualified practitioners are aware about the signs, symptoms, and symptomatic treatment. They know where to refer for diagnosis and treatment. However, the existing referral system was found to be one of the causes that could be a hindrance for achieving expected TB control success in urban slums. This is because, in the study area, the referral system mainly depends on existing informal relationship among the health service providers and also between health service providers and community people. This sometimes leads to referring patients to wrong service providers. NGOs and government hospitals are providing correct treatment but usually patients go to drug sellers first when they have early symptoms. So it all depends on the first point of contact where they refer their patient for diagnosis and treatment. If they are not referring correctly then NGOs and government hospitals lose these patients. So a systematic and formal referral system is a key to achieve broader coverage of TB treatment. Formal network of partnership can also help to scale up knowledge of first point of contact through vigorous and periodic training. It has been evident from the study that health service providers who received training from NGOs and government have more knowledge than other service providers and can be instrumental in identifying and referring patients. The mechanisms of TB programs in urban areas of Bangladesh should seriously consider arranging regular training and monitoring health workforce, setting up formal partnership among formal and informal health service providers and generate information that policy-makers could use to scale up the TB control program. Given the burden of tuberculosis in the urban slum, TB control should continue to remain a priority for coming years.

\section{Acknowledgements}

We would like to thank The Global Fund to fight AIDS, Tuberculosis and Malaria (GFATM) for providing financial support to James P Grant School of Public Health, BRAC University to conduct this research. We also thank Health Nutrition \& Population Programme of BRAC for providing technical support throughout the study.

\section{REFERENCES}

Ahmed, N. U., Alam, M. M., Sultana, F., Sayeed, S. N., Pressman, A. M., \& Powers, M. B. (2006). Reaching the unreachable: Barriers of the poorest to accessing NGO healthcare services in Bangladesh. Journal of Health Populnutr, 24, 457-465.

Cases in Global Health Delivery (2011). Tuberculosis in Dhaka: BRAC's urban TB program.

Cavalcante, S. C., Soares, E. C. C., Pacheco, A. G. F., Chaisson, R. E., Durovni, B., \& the DOTS Expansion Team (2007). Community DOT for tuberculosis in a Brazilian favela: comparison with a clinic model. The International Journal of Tuberculosis and Lung Disease, 11, 544-548.

Croft, R. A., \& Croft, R. P. (1998). Expenditure and loss of income incurred by tuberculosis patients before reaching effective treatment in Bangladesh. The International Journal of Tuberculosis and Lung Disease, 2, 252-253.

David, A. M., Mercado, S. P., Becker, D., Edmundo, K., \& Mugisha, F. (2007). The prevention and control of HIV/AIDS, TB and vectorborne diseases in informal settlements: Challenges, opportunities and insights. Journal of Urban Health: Bulletin of the New York Academy of Medicine, 84, 65-74.

Floyd, K. (2003). Costs and effectiveness-The impact of economic studies on TB control. Tuberculosis, 83, 65-74.

Harvard Medical School, Brigham and Women's Hospital (2011). Tuberculosis in Dhaka: BRAC's urban TB program.

Hurtig, A. K., Pande, S. B., Baral, S. C., Newell, J., Porter, J. D., \& Bam, D. S. (2002). Health Policy And Planning, 17, 78-98. http://dx.doi.org/10.1093/heapol/17.1.78

Islam, M. A., May, M. A., Ahmed, F., Cash, R. A., \& Ahmed, J. (2011). Making Tuberculosis History. Dhaka: The University Press Limited.

Karim, F. (2009). Gender matters Understanding of access barriers to community-based tuberculosis care in Bangladesh.

Karim, F., Islam, M. A., Chowdhury, A. M. R., Johansson, E., \& Diwan, V. K. (2007). Gender differences in delays in diagnosis and treatment of tuberculosis. Health Policy and Planning, 22, 329-334.

Mushtaq, M. U., Shahid, U., Abdullah, H. M., Saeed, A., Omer, F., Shad, M. A., et al. (2011). Urban-rural inequities in knowledge, attitudes and practices regarding tuberculosis in two districts of Paki- 
stan's Punjab province. International Journal for Equality in Health, 10, 2-7.

Nolan, C. M., Schecter, G., Mase, S. R., Jereb, J., Navin, T. R., Posey, D. L., et al. (2007). Evaluation of tuberculosis program services for Burmese refugees in Thailand resettling to the United States, June 2007.

Patel, R. B., \& Burke, T. F. (2009). Urbanization-An emerging humanitarian disaster. The New England Journal of Medicine, 361, 741-743. http://dx.doi.org/10.1056/NEJMp0810878

Public Health Watch (2006). TB policy in Bangladesh.

Rahman, K. A., Kamsrichan, W., \& Keiwkarnka, B. (2008). Factors related to acceptance of tuberculosis case detection among urban slum population in Mohammadpur, Dhaka city corporation, Bangladesh. Journal of Public Health \& Development, 6, 82-88.

Rashid, S. F. (2009). Strategies to reduce exclusion among populations living in urban slum settlements in Bangladesh. Journal of Health Population Nutrition, 27, 574-586.

Rashid, S. F., Akram, O., \& Standing, H. (2011). The sexual reproductive health care market in Bangladesh: where do poor women go? Reproductive Health Matter, 19, 21-30.

Ravichandran, N. (2004). Tuberculosis control in developing countries: A generalized community health worker based model.

Salim, M. A. H., Declercq, E., Deun, A. V., \& Saki, K. A. R. (2004). Gender differences in tuberculosis: A prevalence survey done in Bangladesh. The International Journal of Tuberculosis and Lung Disease, 8, 952-956.

Salim, M. H., Uplekar, M., Daru, P., Aung, M., Declercq, E., \& Lonnroth, K. (2006). Policy and Practice Turning liabilities into resources: Informal village doctors and tuberculosis control in Bangla- desh. Bulletin of the World Health Organization, 84, 479-484. http://dx.doi.org/10.2471/BLT.05.023929

Shah, N. M., Brieger, W., \& Peters, D. H. (2010). Can interventions improve health services from informal private providers in low and middle-income countries? A comprehensive review of the literature. Health Policy and Planning, 26, 276-285.

Standing, H., Rashid, S. F., \& Akram, O. (2013). Informal markets in sexual and reproductive health services and commodities in rural and urban Bangladesh.

Ullah, A. N. Z., Newell, J. N., Ahmed, J. U., Hyder, M. K. A., \& Islam, A. (2006). Government-NGO collaboration: The case of tuberculosis control in Bangladesh. Government-NGO Collaboration in $T B$ Control.

Vissandjee, B., \& Pal, M. (2007). The socio-cultural challenge in public health interventions: the case of tuberculosis in India. International Journal of Public Health, 52, 199-200.

Wikstrom, G. (2011). Women's perspectives on pathway to diagnosis of pulmonary tuberculosis women voices from community level in Uganda. Nordic School of Public Health.

World Health Organization (2011). Tuberculosis prevention, care and control A practical directory of news advances. Geneva: World Health Organization.

World Health Organization (2008). Global tuberculosis control 2008: surveillance, planning, financing. Geneva: World Health Organization.

World Health Organization (2007). Tuberculosis Prevalence Surveys: A handbook. Geneva: World Health Organization.

World Health Organization (2004). Tuberculosis and health sector reforms in Bangladesh. Geneva: World Health Organization. 